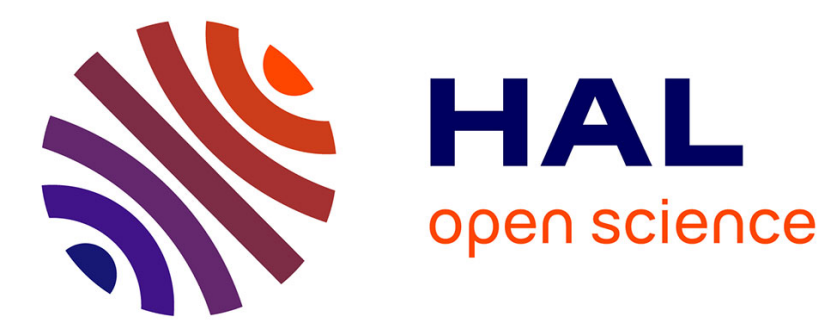

\title{
Identifiability and algebraic identification of time delay systems \\ Lotfi Belkoura
}

\section{To cite this version:}

Lotfi Belkoura. Identifiability and algebraic identification of time delay systems. Ifac Workshop on Time delay Systems, 2010, Prague, Czech Republic. inria-00504943

\section{HAL Id: inria-00504943 https://hal.inria.fr/inria-00504943}

Submitted on 22 Jul 2010

HAL is a multi-disciplinary open access archive for the deposit and dissemination of scientific research documents, whether they are published or not. The documents may come from teaching and research institutions in France or abroad, or from public or private research centers.
L'archive ouverte pluridisciplinaire HAL, est destinée au dépôt et à la diffusion de documents scientifiques de niveau recherche, publiés ou non, émanant des établissements d'enseignement et de recherche français ou étrangers, des laboratoires publics ou privés. 


\title{
Identifiability and algebraic identification of time delay systems *
}

\author{
Lotfi Belkoura \\ LAGIS(FRE 3303 CNRS) 83 Alien Project (INRIA LNE), \\ Universite Lille Nord de France
}

\begin{abstract}
Identifiability and algebraic identification of time delay systems are investigated in this paper. Identifiability results are first presented for linear delay systems described by convolution equations. On-line algorithms are next proposed for both parameters and delay estimation. Based on a distributional technique, these algorithms enable an algebraic and simultaneous estimation by solving a generalized eigenvalue problem. Simulation studies with noisy data and experimental results show the performance of the proposed approach.
\end{abstract}

Keywords: Time delay system, Identifiability, Identification.

\section{INTRODUCTION}

The real time delay identification is one of the most crucial open problems in the field of delay systems (see, e.g., Richard [2003]), and several on line estimation methods have been suggested in the literature for the identification of delay. While the most popular technique of Pade approximation is limited by the range of validity of the approximation, most of the other approaches (see, e.g., Gomez et al. [2007], Drakunov et al. [2006] for adaptive techniques or Ren et al. [2005] for a modified least squares technique) generally suffer from poor speed performance. Recent developments in Belkoura et al. [2009] have considered the on line identification of delay systems with particular (structured) inputs, and this paper extends the identification problem to more general input-output trajectories.

In an identification problem, and prior to the design off efficient estimation algorithms, the question arises as to in which sense an input/output description of the process, if any, is unique. An identifiability analysis is then required to ensure uniqueness off the proposed description. This aspect is also addressed in this paper for linear systems, where identifiability conditions, which can be formulated in terms of controllability of time delay systems, are presented for a general class of systems with discrete and distributed delays.

In the proposed continuous time framework, the timedelay is not restricted to be a multiple of some sampling interval, and exact and non asymptotic formulations are obtained for both parameters and delay estimations. Although the parameter estimation technique is still inspired from the fast identification techniques that were proposed Fliess M. [2003] for linear, delay free and finite-dimensional models, this paper considers a distributional approach in

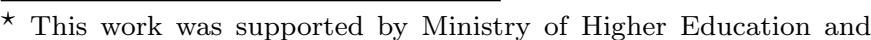
Research, Nord-Pas de Calais Regional Council and FEDER through the 'Contrat de Projets Etat Region' (CPER) 2007-2013.
}

which the cancellation techniques are reduced to simple multiplication by appropriate functions.

The paper is organized as follows. Section 2 presents sufficient identifiability conditions for time delay systems, and Section 3 presents the estimation methods for parameters and delay identification in case of structured entries. The unstructured case, where the cancellation method can not be applied is considered in Section 4. In this section, the problem of initial conditions that allows experiments to start from an arbitrary starting point is also addressed. Most of our developments are illustrated on a first or second order processes, although extension to higher order systems is generally straightforward. The following subsection presents the general framework and specific tools that are required in the subsequent developments.

\section{The distribution Framework}

Throughout this paper, functions will be considered through the distributions they define, i.e. as continuous linear functionals on the space $\mathcal{D}$ of $C^{\infty}$-functions having compact support in $[0, \infty)$. This framework allows the definition of the Dirac distribution $u=\delta$ and its derivative $u=\dot{\delta}$ as $\langle u, \varphi\rangle=\varphi(0)$ and $\langle u, \varphi\rangle=-\dot{\varphi}(0), \varphi \in \mathcal{D}$ respectively. More generally, every distribution is indefinitely differentiable, and if $u$ is a continuous function except at a point $a$, its distributional derivative writes:

$$
u^{(1)}=\frac{d u}{d t}+\sigma_{a} \delta_{a}
$$

where $\delta_{a}:=\delta(t-a), \sigma_{a}:=u\left(t_{a}+\right)-u\left(t_{a}-\right)$, and $\frac{d u}{d t}$ stands for the distribution stemming from the usual derivative (function) of $u$ defined almost everywhere. Note that with $a=0$, this result, and its extension to higher order derivation, is nothing but the analog part of the familiar Laplace transform $\mathcal{L}(\dot{y})=s y(s)-y_{0}$.

We proceed this introductory section with some wellknown definitions and results from the convolution products, and as usual, denote $\mathcal{D}_{+}^{\prime}$ the space of distributions 
with support contained in $[0, \infty)$. It is an algebra with respect to convolution with identity $\delta$. For $u, v \in \mathcal{D}_{+}^{\prime}$, this product is defined as $\langle u * v, \varphi\rangle=\langle u(x) \cdot v(y), \varphi(x+y)\rangle$, and can be identified with the familiar convolution product $(u * v)(t)=\int_{0}^{\infty} u(\theta) v(t-\theta) d \theta$ in case of locally bounded functions $u$ and $v$. Derivation, integration and translation can also be defined from the convolutions $\dot{u}=\dot{\delta} *$ $u, \quad \int u=H * u, \quad u(t-\tau)=\delta_{\tau} * u$, where $H$ is the familiar Heaviside step function. We also recall the following well known property:

$$
u(t) * v(t-\tau)=u(t-\tau) * v(t)=\delta_{\tau} * u * v .
$$

As for the supports, one has for $u, v \in \mathcal{D}_{+}^{\prime}$ :

$$
\operatorname{supp} u * v \subset \operatorname{supp} u+\operatorname{supp} v \text {, }
$$

where the sum in the right hand side is defined by

$$
A+B=\{x+y ; x \in A, y \in B\}
$$

The specific need for the distributional framework also lies in its ability to cancel the singular terms simply by means of multiplication with some appropriate functions. Multiplication of two distributions (say $\alpha$ and $u$ ) always make sense when at least one of the two terms (say $\alpha$ ) is a smooth function, and the cancellation procedure presented in this paper will be derived from the following general Theorem:

Theorem 1. Schwartz [1966] If $u$ has a compact support $K$ and is of order $r$ (necessarily finite) ${ }^{1}, \alpha u=0$ whenever $\alpha$ and its derivatives of order $\leq r$ vanish on $K$.

Particularly, one has for the singular Dirac distributions:

$$
\begin{gathered}
\alpha \delta_{\tau}=\alpha(\tau) \delta_{\tau}, \\
\alpha \delta_{\tau}^{(r)}=0 \quad \forall \alpha \text { s.t. } \alpha^{(k)}(\tau)=0, \quad k=0, \ldots, r .
\end{gathered}
$$

Finally, with no danger of confusion, we shall sometimes denote $u(s), s \in \mathbb{C}$, the Laplace transform of $u$.

\section{IDENTIFIABILITY ANALYSIS}

A standard approach for identification of systems implies that the structure of the system is known and the problem is in finding the values of parameters (including the delays) involved in the set of equations describing the process. The ability to ensure this objective is typically referred to as parameter identifiability. First results on identifiability for delay differential equations can be found in S. Nakagiri [1995], Lunel [1997, 2001]. However, these results are limited to the homogeneous case (no forcing term) and use a spectral approach involving infinite dimensional spectrum. The approach used in Belkoura [2005] extends the identifiability analysis to more general systems described by convolution equations of the form:

$$
R * w=0, \quad R=[P,-Q], \quad w=\left[\begin{array}{c}
y \\
u
\end{array}\right],
$$

where $P(n \times n)$ and $Q(n \times q))$ are matrices with entries in the space $\mathcal{E}^{\prime}$ of distributions with compact support. Equation (7) correspond to a behavioral approach of systems described by convolutional equations (see Vettori P.

1 The order of a distribution $u$ is the smallest integer $m$ such that: $\exists C>0$ such that for any smooth function $\varphi$, one has $|\langle u, \varphi\rangle| \leq$ $C \sup \left\|\varphi^{(i)}\right\|_{\infty}$ (for example, $\delta^{(r)}+$ lower order terms is of order $0 \leq i \leq m$

$r)$.
[2000]). Here, $R(s)$, the Laplace transform of $R$, provides a kernel representation of the behavior $\mathcal{B}$ which consists in the set $\tilde{w}$ of all admissible trajectories in the space of $C^{\infty}(\mathbb{R}, \mathbb{R})$ functions, and $\tilde{w} \in \mathcal{B}=\operatorname{ker}_{\mathcal{E}} R(s)$.

The concept of identifiability is based on the comparison of the original system and its associated reference model governed by (7) and in which $R, P, Q$ and $y$ are replaced by $\hat{R}, \hat{P}, \hat{Q}$ and $\hat{y}$ respectively. System (7) is therefore said to be identifiable if there exists a control $u$ such that the output identity $\hat{y}=y$ results in $\hat{R}=R$, which means uniqueness of the matrix coefficients as well as that of the delays.

For most practical cases, and provided a sufficiently rich input signal, identifiability of (7) reduces to

(1) $\operatorname{rank} R(s)=n, s \in \mathbb{C}$,

(2) conv $\operatorname{det} P=n \operatorname{conv} R$.

where conv $R$ denote the smallest closed interval that contains the support of $R$ (i.e. the convex hull of $\operatorname{supp} R$ ), $\operatorname{det} P$ is the determinant with respect to the convolution product, and $n$ conv $R$ stands for the sum of $n$ terms as defined in (4). These conditions are closely linked to the property of approximate controllability in the sense that the reachable space is dense in the state space Yamamoto [1989].

The following example Belkoura [2005] shows the applicability of the previous result to systems with distributed delays. Consider the multivariable delay system

$$
\begin{aligned}
& \dot{x}_{1}(t)=x_{1}(t)+\int_{-1}^{0} x_{2}(t+\tau) d \tau \\
& \dot{x}_{2}(t)=x_{1}(t-1)+x_{2}(t)+\int_{-1}^{0} u(t+\tau)
\end{aligned}
$$

and denote $\pi(t)=H(t)-H(t-1)$, with $H$ the Heaviside function. Here, $\operatorname{supp} \pi=[0,1]$ and some simple manipulations show that system (8) admits a kernel representation $R * \omega=0$ with $\omega=\left(x_{1}, x_{2}, u\right)^{T}$ and

$$
R=[P,-Q]=\left[\begin{array}{cc|c}
\delta^{\prime}-\delta & -\pi & 0 \\
-\delta_{1} & \delta^{\prime}-\delta & -\pi
\end{array}\right]
$$

Clearly, $\operatorname{conv} R=[0,1]$ while $\operatorname{det} P=\delta^{\prime \prime}-2 \delta^{\prime}+\delta-\delta_{1} * \pi$, from which one easily gets conv $\operatorname{det} P=[0,2]$, so condition (2) of is satisfied. On the other hand,

$$
R(s)=\left[\begin{array}{ccc}
s-1 & -\left(e^{-s}-1\right) / s & 0 \\
-e^{-s} & s-1 & -\left(e^{-s}-1\right) / s
\end{array}\right],
$$

and the determinant formed by the second and third column of $R(s)$ is nonzero for $s \neq 0$, and for $s=0$, the first and second column of $R(0)$ form a non singular matrix. Hence condition (1) is also satisfied and system (8) is identifiable.

In the case of distributed delays, the major limitation of the previous approach is the need of the largest delay involved in (7). In return for more restrictive models with lumped and commensurate delays of the form

$$
\dot{x}(t)=\sum_{i=0}^{r} A_{i} x(t-i . h)+B_{i} u(t-i . h),
$$

a simpler identifiability result which no longer requires the assumption of an a priori known memory length is obtained in Belkoura L. [2002]. It can be expressed in terms 
of weak controllability, concept introduced in Morse [1976] for systems over rings, through the rank condition (over the ring $R[\nabla])$ :

$$
\begin{aligned}
& \operatorname{rank}\left[B(\nabla), \ldots, A^{n-1}(\nabla) B(\nabla)\right]=n, \\
& A(\nabla)=\sum_{i=0}^{r} A_{i} \nabla^{i}, \quad B(\nabla)=\sum_{i=0}^{r} B_{i} \nabla^{i} .
\end{aligned}
$$

Note however that all the previous results are limited to linear and time invariant models. In case of nonlinear delay systems or time dependent delays, general identifiability results are still expected.

\subsection{Sufficiently rich input}

In identification procedures the design of a sufficiently rich input which enforces identifiability is also an important issue. Given a reference model associated to the process under study, one has to know whether equality of the outputs results in that of the transfer functions. Few results are dealing with such issue for time delay systems. In Belkoura L. [2004], Belkoura [2005] the input design is considered in the time domain rather than the frequency domain and the approaches are mainly based on the non smoothness of the input. More precisely, if

$$
\Lambda_{u}=\left\{s_{0}, s_{1}, \ldots, s_{L}, \ldots\right\}
$$

denote the singular support of $u$ (i.e. the set of points in $\mathbb{R}$ having no open neighborhood to which the restriction of $u$ is a $C^{\infty}$ function), the input is required to be sufficiently "discontinuous" in the sense that

$$
\operatorname{rank}\left[U_{0}(D), \ldots, U_{L}(D)\right]=q
$$

where the polynomial matrices $U_{l}(D)$ are formed with the (possible) jump of $u^{(k)}(t)$ for some $k \geq 0$ at $t=s_{l}$ by

$$
\begin{aligned}
& U_{l}(D)=\sum_{i=0}^{k} \sigma_{l}^{k-i} D^{i}, \\
& \sigma_{l}^{k-i}=u^{(k-i)}\left(s_{l}+0\right)-u^{(k-i)}\left(s_{l}-0\right) .
\end{aligned}
$$

On the other hand, "the discontinuity points" $s_{0}, s_{1},$. . should be sufficiently spaced in the general case of distributed delays, although for lumped delays, this constraint (which may constitute a serious drawback in situations where on line procedures are used) can be relaxed using commensurability considerations. The simplest example consists of a piece-wise constant $\mathbb{R}^{q}$-valued function with appropriate discontinuities, although inputs of class $\mathcal{C}^{r}$ for an arbitrary finite integer $r$ can be formed.

While the identifiability analysis of this section is developed for complex systems with possibly distributed delay, most of the subsequent identification algorithms are performed on simple linear and non linear examples for which the present study turn out to be either straightforward or non applicable. The algebraic identification procedures presented in the next sections clearly separate the case of structured entries (that can be annihilated by means of simple algebraic manipulations) from the unstructured ones.

\section{ALGEBRAIC IDENTIFICATION: THE STRUCTURED CASE}

\subsection{Structured signals and their annihilation}

Structured entries have been introduced (see e.g. Fliess M. [2003]) to refer to entities (mainly perturbations) that can be annihilated by means of simple multiplications and derivations. A simple example consists for instance in a load perturbation, modeled by a Heaviside function $\xi(t)=H(t)$, and for which one easily obtains, in the time and operational domain respectively:

$$
t \times \frac{d \xi}{d t}=0, \quad \frac{d}{d s} s \xi(s)=0
$$

This aspect is generalized in the Schwartz's Theorem of the introductory Section, and the identification procedure is based on the idea that unknown terms to be identified and contained in a structured term can be recovered by the above cancellation procedure. This approach is illustrated in the following subsections for a simulation and an experimental case respectively.

\subsection{Application to a single delay identification}

Consider a first order nonlinear system subject to a delayed step input $u(t)=H(t-\tau)$. Such systems, where the delays only appear in the control variables, are most common in practice and this simple example emphasizes that linearity w.r.t. input-outputs is not required.

$$
\dot{y}+a y^{2}=y_{0} \delta+b H(t-\tau),
$$

where $a, b$, and $\tau$ are constant parameters. Note that in the distribution sense, the initial condition term occurs as an impulsive term. The coefficient $a$ is assumed to be known, and first order derivation results in equation (20) which may be canceled, for instance, and by virtue of Schwartz'sTheorem by the polynomial $\alpha=t^{3}-\tau t^{2}$, yielding:

$$
\begin{aligned}
& \ddot{y}+a \dot{z}=y_{0} \dot{\delta}+\gamma_{0} \delta+b \delta_{\tau}, \\
& \left(t^{3}-\tau t^{2}\right)(\ddot{y}+a \dot{z})=0,
\end{aligned}
$$

where for ease of notation we have denoted $z=y^{2}$. As an equality of distributions, equation (21) does not make sense for any $t$ (otherwise we would get from (21) $t=\tau)$. However, integration of (21) results in an equality of functions from which the delay becomes available. To ensure causality, $k \geq 2$ integrations can be used, yielding an explicit and non asymptotic formula of the delay:

$$
\tau=\frac{\int^{(k)} t^{3}(\ddot{y}+a \dot{z})}{\int^{(k)} t^{2}(\ddot{y}+a \dot{z})}, \quad t>\tau,
$$

where the symbol $\int^{(k)}$ stands for iterated integration of order $k$. Note that the integration by part, illustrated in the partial realization scheme of Figure 1 avoid any derivative in the estimation algorithms.

Since from $(5), t^{q}(\ddot{y}+a \dot{z}), q=2,3$ have their support reduced to $\{\tau\}$, both numerator and denominator of (22) are with support within $(\tau, \infty)$, so the delay is clearly not identifiable for $t<\tau$. Nevertheless, the delay estimation may be achieved in a small time interval $(\tau, \tau+\epsilon)$. 


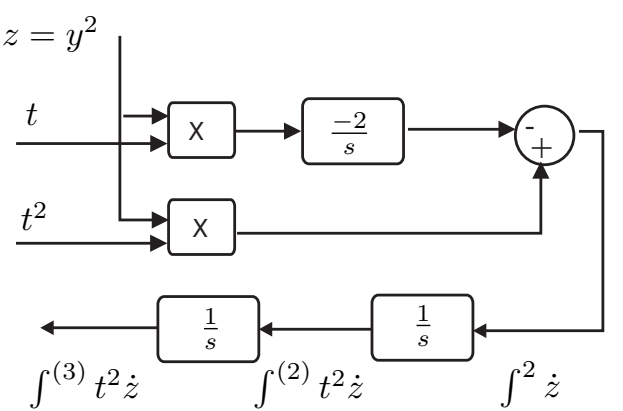

Fig. 1. Realization scheme of $\int^{(k)} t^{2} \dot{z}, \quad k \geq 2$.

Simulation results in a noisy context are depicted in Figure 2 , with $k=4$ integrations, and

$$
(y(0), a, \tau)=(0.3,2.5,0.5) .
$$

In addition to causality requirements, the choice of more than 2 integrations has been used to obtain an additional filtering effect which attenuates the noise from the measurement $y$.
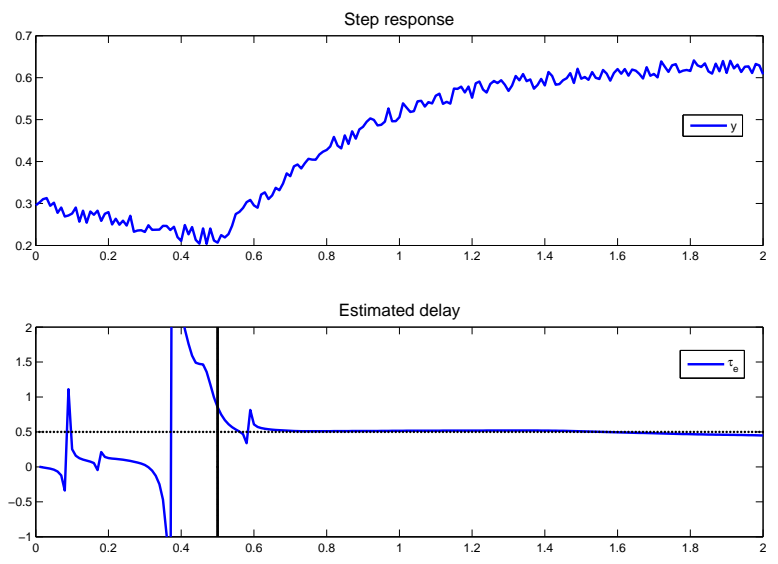

Fig. 2. Trajectory of (19) and delay identification.

\subsection{Application to a simultaneous parameters and delay identification: Experimental example}

In this section, experiments are carried out on a Feedback process PT37 100. This process consists of heating the air flowing in a tube to the desired temperature level. The physical principle which governs the behavior of the this process is the balance of heat energy. The response of the sensor to a change in the heater power is moreover affected by a pure delay, which depends on the velocity of the process and the distance between the point of change and the sensor. In an open loop configuration, the behavior is approximated by a linear system with delayed input. The process is subject to a step input, and a second order model with both unknown parameters and delay is used to describe it.

$$
G(s)=\frac{K e^{-\tau s}}{a_{2} s^{2}+a_{1} s+1},
$$

The candidate function chosen for the annihilation of the structured part is the complex and bounded function $\alpha=$ $\left(1-\lambda e^{-j \omega t}\right)$, with the unknown to be found $\lambda=e^{j \omega \tau} \in \mathbb{C}$, and tunable frequency $\omega$. More explicitly, denoting $e=$ $e^{-j \omega t}$, a derivation of the differential equation derived from
(23), followed by the multiplication by $\alpha$ result respectively in:

$$
\begin{aligned}
& a_{2} y^{(3)}+a_{1} y^{(2)}+y^{(1)}=K \delta_{\tau}, \\
& (1-\lambda e)\left(a_{2} y^{(3)}+a_{1} y^{(2)}+y^{(1)}\right)=0 .
\end{aligned}
$$

We shall focus on the identification of the coefficients $\left\{\lambda, a_{2}, a_{1}\right\}$, and provided a sufficiently large period $2 \pi / \omega$, the delay is deduced from the unique argument $\tau=$ $\arg (\lambda) / \omega$. Due to the terms $\lambda a_{i}, i=1,2,(25)$ is not linear w.r.t. the unknown coefficients, but may be written in the following form:

$$
\left[\left(y^{(3)}, \cdots, y^{(1)}\right)-\lambda\left(e y^{(3)}, \cdots, e y^{(1)}\right)\right]\left(\begin{array}{c}
a_{2} \\
a_{1} \\
1
\end{array}\right)=0 .
$$

As in the previous section, successive integrations transform the equality of singular distributions of (25) into one of continuous functions. Denoting $\Theta=\left(a_{2}, a_{1}, 1\right)^{T}$ the (normalized) vector of parameters, the specific structure of (26) leads to following generalized eigenvalue problem for possibly non square pencils:

$$
(A-\lambda B) \Theta=0,
$$

where, using a Matlab-like notation, the entries of the $m \times 3$ trajectory-dependent matrices $A$ and $B$ are given by

$$
\begin{gathered}
A(i,:)=\int^{(i+2)}\left(y^{(3)}, \cdots, y^{(1)}\right), \quad i=1, \ldots, m \\
B(i,:)=\int^{(i+2)}\left(e y^{(3)}, \cdots, e y^{(1)}\right) \quad i=1, \ldots, m .
\end{gathered}
$$

The implementation of $A(i, j)$ and $B(i, j)$ is performed according to the integration by parts formulas. Therefore, the identification problem has been transformed into the eigenvalue problem (27) in which, at each $t$, the unknown delay $\tau=\arg (\lambda) / \omega$ is derived from one eigenvalue, while the parameters $a_{1}, a_{2}$ are obtained from the corresponding normalized eigenvector. Solving (27) in a noisy context and in the non-square case (i.e. by considering $m>$ 3 lines for $A$ and $B$ ) is generally not an easy task, since (27) "has the awkward feature that most matrices have no eigenvalues at all, whilst for those that do, an infinitesimal perturbation will in general remove them" Wright and Trefethen [2002]. A possible approach can be based on the pseudo-spectra analysis which consists in introducing the $\epsilon$-pseudospectra of (27) (see e.g Wright and Trefethen [2002] and the references therein). However, this approach is not appropriate for on-line perspectives, and we adopt here a simpler technique, based on the a priori stationarity assumption of the unknown parameters. More precisely, the selected parameters correspond to the eigenpair $\left(\lambda_{i}, \Theta_{i}\right)$ of the square pencil (27) that minimizes the norm $\left\|\left(A+\lambda_{i} B\right) \Theta_{i}\right\|$ of the rectangular pencil (i.e. for $m>3)$.

As in the single delay estimation problem of the previous section, it can be easily shown that matrices $A$ and $B$ are continuous matrix functions with support within $(\tau, \infty)$, which means that the delay and parameters are not identifiable for $t<\tau$. Moreover, and although the approach is non asymptotic, this continuity can make the estimation problem sensitive to noise and neglected dynamics in the vicinity of $\tau$. Unlike noise-free contexts or reduced order identification problems such as the delay 
estimation in the previous section, it is clear a minimum amount of trajectories information is required here to obtain a consistent and relevant eigenvalue problem.

Figure 3 (top) shows the experimental response as well as the simulated trajectories based on the identified delay and parameters (bottom). Although the convergence algorithm is clear, the time history of the identified parameters reflects a singularity of the eigenvalue problem in the vicinity of $t=0.8 \mathrm{~s}$. The implementation of (28) was converted to discrete-time, assuming a sampling period of $50 \mathrm{msec}$, and resolution of (27) has been made using the polyeig function of the Matlab software. Taking into
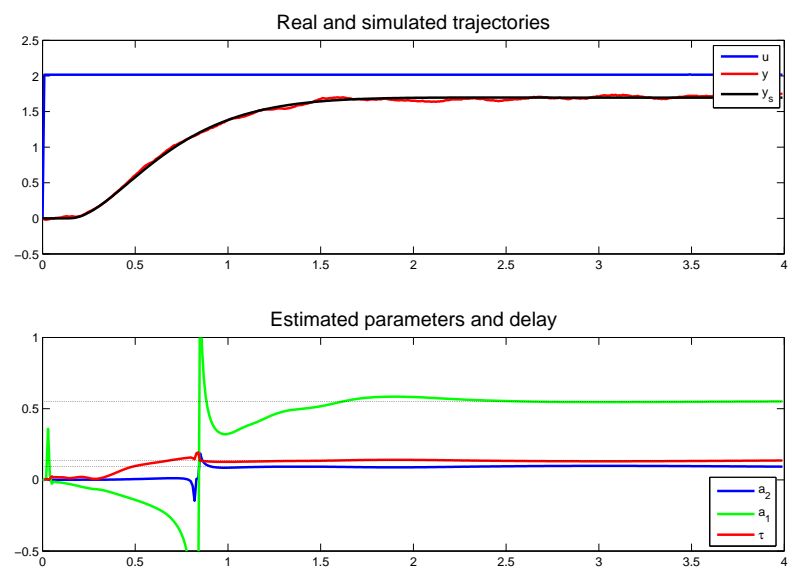

Fig. 3. Experimental and simulated trajectories (top). Estimated delay and parameters (bottom)

account the static gain $K$ estimated by other means, the identified second order model for the process reads:

$$
G_{1}(s) \approx \frac{0.84 e^{-0.13 s}}{0.09 s^{2}+0.55 s+1} .
$$

\section{ALGEBRAIC IDENTIFICATION: THE UNSTRUCTURED CASE}

When facing arbitrary inputs, the above annihilation procedure no longer applies, but algebraic estimation results can be still obtained by mean of an approach combining multiplication an cross convolution, as described below. Provided the system is initially at rest (null initial condition), state delay may also be identified.

\subsection{The cross convolution approach}

We first focus on a single delay identification regardless of any process dynamics. When considered on the whole real line, a delay between two functions $a(t)$ and $b(t)$ reads as in (30) and leads to (31) once multiplied by any deviated known function $\alpha(t-\tau)$.

$$
\begin{aligned}
a(t) & =b(t-\tau), \\
\alpha(t-\tau) a(t) & =(\alpha b)(t-\tau) .
\end{aligned}
$$

Using (2), a convolution product derived from these two relations results in equation (33) with no deviated argument in the original functions $a$ and $b$.

$$
\begin{aligned}
& {[\alpha(t-\tau) a(t)] * b(t-\tau)=a(t) *(\alpha b)(t-\tau), } \\
\Rightarrow & {[\alpha(t-\tau) a(t)] * b(t)=a(t) *(\alpha b)(t) . }
\end{aligned}
$$

If the adopted function $\alpha(t-\tau)$ admits an expansion separating its arguments $t$ and $\tau$, i.e.:

$$
\alpha(t-\tau)=\sum_{i \text { finite }} \lambda_{i}(t) \mu_{i}(\tau)
$$

for some known functions $\lambda$ and $\mu$, then an algebraic relation is obtained allowing a non asymptotic and explicit delay formulation, as illustrated in the simple following examples:

$$
\begin{array}{r}
\alpha(t)=t \Rightarrow \tau=\frac{t a * b-a * t b}{b * a} \\
\alpha(t)=e^{\gamma t} \Rightarrow e^{\gamma \tau}=\frac{b * e^{\gamma t} a}{a * e^{\gamma t} b}
\end{array}
$$

Provided the involved convolution products are well defined, this delay formula holds for all nonzero values of their denominators. More precisely, if the signal $b$ consists in measurements on $(0, \infty)$, then supp $a \subset(\tau, \infty)$ and hence, by virtue of (3), both numerator and denominator of $(35)$ and $(36)$ have their support within $(\tau, \infty)$. Therefore, the delay is not identifiable for $t<\tau$. However, as in the finite dimensional case (see, e.g., Fliess and SiraRamirez [2007]), the input signal $b$ being used in this algebraic approach does not necessarily exhibit the classical "persistency of excitation" requirement. Although a local loss of identifiability may occur due to the zero crossing of the denominator, only non trivial trajectories are required.

Once again, when facing derivatives, one of the nice features of multiplication by polynomial or exponential functions lies in the ability to use simple integration by parts formulas to avoid any derivation in the identification algorithm. The next paragraph illustrates the time lag identification for the delayed integrator:

\subsection{Application to a delay identification}

Consider the following linear first order process with delayed input:

$$
\dot{y}+y=k u(t-\tau)
$$

which correspond to the formulation (30) with $a=\dot{y}+y$ and $b=k u$. In order to avoid multiplications by unbounded functions (polynomials), and hence the amplification of noise and neglected dynamics, a decaying exponential functions is considered and equation (36) reads:

$$
\lambda=\frac{u * e^{\gamma t}(\dot{y}+y)}{(\dot{y}+y) * e^{\gamma t} u}
$$

where we have denoted $\lambda=e^{-\gamma \tau}$ for some tunable positive parameter $\gamma$. Note that the static gain value $k$ is not required nor identified. Denoting $e(t)=e^{-\gamma t}$ and taking into account the integration by parts formula, $\int e \dot{y}=e y+$ $\gamma \int e y$, on gets:

$$
\lambda=\frac{e y * u+(1+\gamma) \int(e y * u)}{e u * y+\int e u * y},
$$

while the delay is obtained from $\tau=\log (\lambda) / \gamma$. For this simple example, and since only a constant delay has to be identified, an additional step considering the integral of the square of equation (39) (i.e. $\int(39)^{2}$ ) avoids the 
possible singularities resulting from the zero crossing of the denominator $e u * y$. This finally results in the delay estimation:

$$
\lambda=\left[\frac{\int_{0}^{t}\left[u * e y+\gamma \int_{0}^{\theta}(u * e y)\right]^{2} d \theta}{\int_{0}^{t}\left[e u * y+\int_{0}^{\theta}(e u * y)\right]^{2} d \theta}\right]^{\frac{1}{2}} .
$$

A simulation result with noisy data is depicted in Figure 4, for an input $u(t)=\sin (t) \cdot(0.2+\sin (0.2 t)), \gamma=0.2$, and a delay $\tau=0.3 \mathrm{~s}$. The simulation step size has been fixed to $0.05 \mathrm{~s}$, and the integrals involved in the convolutions have been approximated by simple sums.
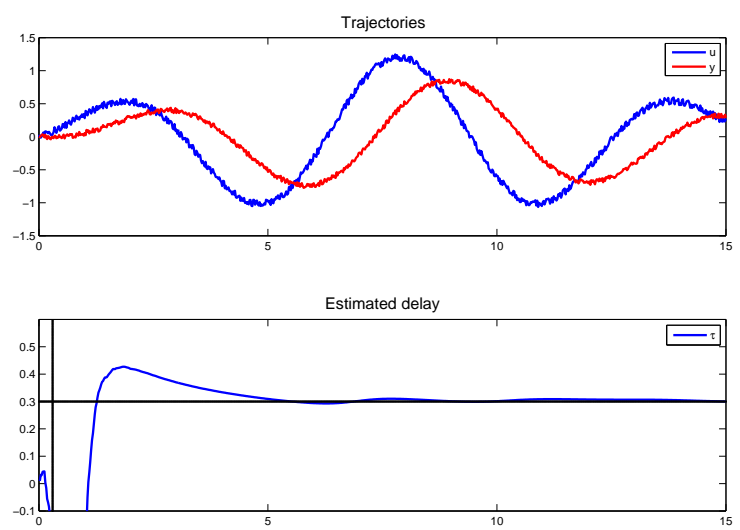

Fig. 4. Trajectories and estimated delay of Eq.(37).

\subsection{Experimental results (continued)}

The above delay estimation procedure is considered in this section for signals $a$ and $b$ describing heating process of Section 3.3. The system is governed by:

$$
\sum a_{i} y^{(i)}(t)=K u(t-\tau)
$$

whose left and right hand sides can be identified with the functions $a$ and $b$ of the previous section (equation 30) as:

$$
a=\sum a_{i} y^{(i)}, \quad b=K \varepsilon .
$$

The exponential function $\alpha(t)=e^{-\gamma t}$, with $\gamma>0$ is adopted for the multiplication step, leading to a reformulation of equation (33) as:

$$
\lambda \sum a_{i}\left[u *\left(e^{-\gamma t} y^{(i)}\right)\right]=\sum a_{i}\left[y^{(i)} *\left(e^{-\gamma t} u\right)\right],
$$

where the unknown delay to be identified is contained in the new unknown term $\lambda=e^{-\gamma \tau}$. Note that the process gain $K$ has been removed by this procedure and will not be estimated. Clearly, equation (43) is non causal and non linear with respect to the delay and parameters $a_{i}$. The use of successive filters $h_{j}(s)$ of relative degree $>2$ allow one the one hand, to ensure causal relations from (43) and on the other hand, to obtain enough equations for a simultaneous estimation of both parameters and delay. Recalling $\Theta=\left(a_{2}, a_{1}, 1\right)^{T}$ the (normalized) vector of parameters, this results in the following estimation problem:

$$
\begin{aligned}
& (V-\lambda W) \Theta=0, \\
& V_{i j}=s^{i} h_{j}(s)\left[y * e^{-\gamma t} u\right](s), \\
& W_{i j}=(s+\gamma)^{i} h_{j}(s)\left[e^{-\gamma t} y * u\right](s),
\end{aligned}
$$

where the realization of each term of the filtering of equation (43), avoiding any measurement's derivative, is based on the familiar property of Laplace transform, $\mathcal{L}\left[e^{\gamma t} f(t)\right](s)=F(s-\gamma)$. In the available data $V_{i j}$ and $W_{i j}$, the notation $[a * b](s)$ emphasizes the measurement based convolution products subject to the filtering procedure. It is worth noticing that in case of a structured input $u$ admitting a simple operational description (for instance a step $\mathrm{u}(\mathrm{s})=1 / \mathrm{s})$, the realization of the entries of $V$ and $W$ reduces to simple filtering of $y$ and $e^{-\gamma t} y$ as:

$$
\begin{aligned}
& V_{i j}=v_{i j}(s) y(s), \quad v_{i j}(s)=s^{i} h_{j}(s) u(s+\gamma), \\
& W_{i j}=w_{i j}(s) y(s+\gamma), \quad w_{i j}(s)=(s+\gamma)^{i} h_{j}(s) u(s)
\end{aligned}
$$

Figure 5 shows the convergence of the estimated parameters in case of a step input, where we adopted simple integrations for the filters (i.e. $h_{j}(s)=1 / s^{j+2}$ ). Taking into account the static gain $K$ estimated by other means, we recover closed results compared to the transfer function (29) estimated in Section 3.3:

$$
G_{2}(s) \approx \frac{0.84 e^{-0.136 s}}{0.1 s^{2}+0.55 s+1}
$$

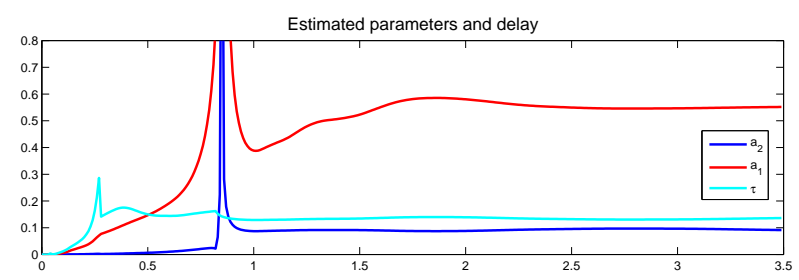

Fig. 5. Estimated delay and parameters of the heat process

\subsection{Systems with general delayed input or state and arbitrary initial conditions}

In a general case where measurements start from non zero rest position, equation (30) must be modified in order to take into account the initial condition term. Unlike ordinary differential equations, such an initial term is infinite dimensional. In the distribution framework, the exact expression is obtained via a truncation mapping (one can refer to Yamamoto [1989] for more details). To illustrate this result, the simple case $\dot{y}=k \varepsilon(t-\tau)$, where $\varepsilon=y_{c}-y$ and $y_{c}$ is a given reference trajectory is rewritten:

$$
\begin{aligned}
& \dot{y}=\varepsilon(t-\tau)+\psi_{0}, \\
& \psi_{0}=y(0) \delta+\varepsilon(\theta), \quad \theta \in(-\tau, 0),
\end{aligned}
$$

and we recover here the "real initial datum" $\psi_{0}$ consisting in an element of $\mathbb{R} \times L^{2}(0, h: \mathbb{R})$ as one can find in A. Bensoussan and Mitter [1992].

In this context, neither the annihilation procedure nor the cross convolutions approach can be applied unless some approximations are done. In the first case, one can consider 
a polynomial approximation of the initial condition term and use the annihilation procedure of Section 3.1 to cancel such an unknown term. In the second case, and even if $\psi_{0}$ consists in a compact support function, the unknown additional terms involved in the cross convolution are not compact supported nor vanishing. One can therefore consider the annihilation of this IC term by means of a premultiplication of (48) by an "almost zero" function on the interval $(0, \tau)$ as shown in Belkoura et al. [2007]. The rest of this section is devoted to an alternative convolution based approach one can find in Belkoura et al. [2008]. Let us consider the delayed integrator rewritten as:

$$
\dot{y}=k u(t-\tau)+\psi_{0} .
$$

Let us assume that we are given an upper bound $\bar{\tau}$ of the unknown delay, and consider $T>\bar{\tau}$ and a smooth function $\alpha$ such that

$$
\operatorname{supp} \alpha \subset(\bar{\tau}, T-\bar{\tau}) \subset(0, T) .
$$

Typically, $\alpha$ may be viewed as an element of the test functions used in the distribution framework and one has for an arbitrary integer $p, \operatorname{supp} \alpha^{(p)} * \psi_{0} \subset(0, T+\tau)$, so that for $t>T+\tau$, one can write from (50):

$$
\int_{0}^{T} \alpha^{(p+1)} * y=k \int_{0}^{T} \alpha^{(p)}(\bullet-\tau) * u .
$$

In the next step, the shifted candidate function $\alpha$ and its derivatives are replaced by their Fourier series approximations of order $n$, yielding

$$
\begin{aligned}
\alpha(\theta) & \approx \sum_{q=-n}^{n} c_{q} e^{j q \omega \theta}, \\
\alpha^{(p)}(\theta-\tau) & \approx \sum_{q=-n}^{n} \lambda^{q} c_{p, q} e^{j q \omega \theta},
\end{aligned}
$$

where we have denoted $c_{p, q}=(j q \omega)^{p} c_{q}, \omega=2 \pi / T$, and the unknown delay dependent coefficient $\lambda=e^{-j \omega \tau}$. Using these approximations in (52) for different $p$, we end up with the following generalized eigenvalue formulation,

$$
\begin{gathered}
\sum_{q=-n}^{n} A_{q} \lambda^{q}\left(\begin{array}{c}
k \\
1
\end{array}\right)=0 \\
A_{q, q \neq 0}=\left(\begin{array}{cc}
b_{q, 0} & 0 \\
\cdots & \cdots \\
b_{q, n_{p}} & 0
\end{array}\right), A_{0}=\left(\begin{array}{cc}
b_{0,0} & -a_{0} \\
\cdots & \cdots \\
b_{0, n_{p}} & -a_{n_{p}}
\end{array}\right), \\
a_{p}(y)=\int_{0}^{T} \alpha^{(p+1)}(\theta) y(t-\theta) d \theta \\
b_{q, p}(u)=\int_{0}^{T} c_{p, q} e^{j q \omega \theta} u(t-\theta) d \theta .
\end{gathered}
$$

A simulation result is shown in Figure 6, where the sliding window size $\mathrm{T}$ has been fixed to $5 s, n=3$ and a choice of $\alpha$ fixed to $\alpha=\sin ^{6}(\omega t / 2)$ on the interval $(0, T)$, with the a priori assumption $T=5>>$. Note that a good estimation is obtained for $t<T$, and the small and local deviations one can observe result from the specific trajectories for which the eigenvalue problem (55) becomes nearly singular. It is worth noticing that compared to Pade based approaches, the ability to identify from bounded sets $(t, t-T)$ of measurements make it possible to extend the parameters estimations to non stationary cases with slowly time varying coefficients. In Figure 7, these estimations are shown for the delayed integrator with slowly time varying gain $k(t)=2(1+.2 \sin (.03 t))$ and delay $\tau(t)=0.3(1-$ $0.8 \sin (.06 t) \cos (.01 t))$.
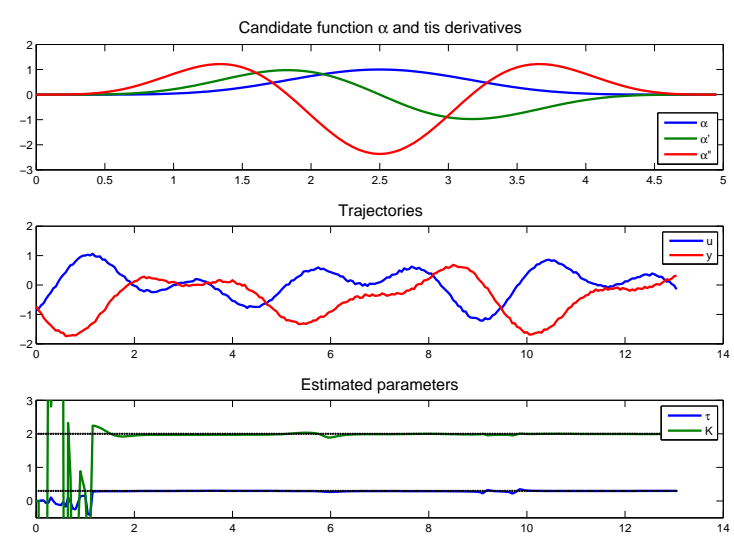

Fig. 6. Candidate function $\alpha$, trajectories and estimated parameters $k, \tau$ of Eq. (48).
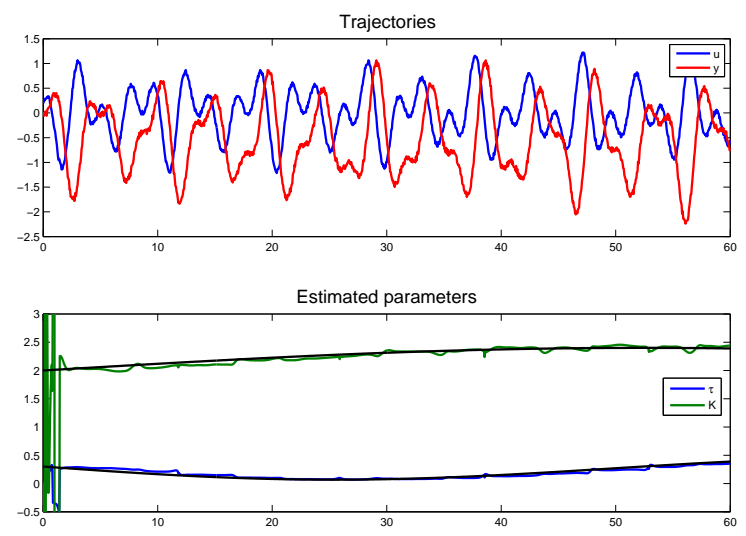

Fig. 7. Trajectories and estimated parameters $k(t), \tau(t)$ of Eq. (48) in case of slowly time varying coefficients.

\section{CONCLUSION}

We have presented sufficient conditions for the identifiability of a general class of systems described by convolution equations. More specifically, for delay differential systems, it is shown how the identifiability property can be formulated in terms of approximate controllability or weak controllability, depending on the available models.

The second part of this paper has presented an algebraic method for the identification of delay systems based on both structured inputs and arbitrary input-output trajectories. The ability of identification on bounded sets of measurements also allows us to extend the estimation problem to slowly time varying parameters and delay. Extensions to the identification in the multivariable and multidelay cases, rigorous proofs for non stationary processes, a well as a deeper study of the eigenvalue problem singularities are open problems under investigation.

\section{REFERENCES}

A. Bensoussan, G. Da Prato, M.D. and Mitter, S. (1992). Representation and control of infinite dimensionnal systems, Vol. I. Birkäuser, Boston.

Belkoura, L. (2005). Identifiability of systems described by convolution equations. Automatica, 41, 505-512.

Belkoura, L., Richard, J.P., and Fliess, M. (2007). Real time identification of delay systems. IFAC Workshop on 
Time Delay Systems, TDS'0\%, Nantes, France, september 17-19.

Belkoura, L., Richard, J.P., and Fliess, M. (2008). A convolution approach for delay systems identification. 17th IFAC World Congress, Seoul, Corea.

Belkoura, L., Richard, J.P., and Fliess, M. (2009). Parameters estimation of systems with delayed and structured entries. Automatica, 45, 5, 1117-1125.

Belkoura L., O.Y. (2002). Identifiability analysis of linear delay-differential systems. IMA J. of Mathematical Control and Information, 19, 73-81.

Belkoura L., Dambrine M., O.Y.R.J.P. (2004). Identifiability and identification of linear systems with delays. Springer, LNCSE Advances in Time Delay Systems, 38, $123-135$.

Drakunov, S., Perruquetti, W., Richard, J.P., and Belkoura, L. (2006). Delay identification in time-delay systems using variable structure observers. Annual Reviews in Control, 143-158.

Fliess, M. and Sira-Ramirez, H. (2007). Closed-loop parametric identification for continuous-time linear systems via new algebraic techniques. In H.G..L. Wang (ed.), Continuous-Time Model Identification from Sampled Data. Springer, http: //hal.inria.fr/ inria-00114958.

Fliess M., S.R.H. (2003). An algebraic framework for linear identification. ESAIM Control, Optimization and Calculus of Variations, 9.

Gomez, O., Orlov, Y., and Kolmanovsky, I. (2007). Online identification of siso linear time-delay systems from output measurements. Automatica, 43, 12, 2060-2069.

Lunel, S. (1997). Identification problems in functionnal differential equations. Proc. 36th conf. on Decision and Control, 4409-4413.

Lunel, S. (2001). Parameter identifiability of differential delay equations. Int J. of Adapt. Control Signal Process., 15, 655-678.

Morse, A. (1976). Ring models for delay differential systems. Automatica, 12, 529-531.

Ren, X., Rad, A., Chan, P., and Lo, W. (2005). On line identification of continuous-time systems with unknown time delay. Ieee Tac, 50-9, 1418-1422.

Richard, J. (2003). Time-delay systems: an overview of some recent advances and open problems. Automatica, 39, 1667-1694.

S. Nakagiri, M.Y. (1995). Unique identification of coefficient matrices, time delay and initial functions of functionnal differential equations. Journal of Mathematical Systems, Estimation and Control, 5, No 3, 323-344.

Schwartz, L. (1966). Théorie des distributions. Hermann, Paris.

Vettori P., Z.S. (2000). Controllability of systems described by convolutionnal or delay-differential equations. SIAM Journal of Control and Optimization, 39, No 3, 728-756.

Wright, T. and Trefethen, L. (2002). Pseudospectra of rectangular matrices. IMA J. of Numer. Anal., 501519.

Yamamoto, Y. (1989). Reachability of a class of infinitedimensional linear systems: An external approach with application to general neutral systems. SIAM J. of Control and Optimization, 27, 217-234. 